\title{
Congestive gastropathy and Helicobacter pylori: an endoscopic and morphometric study
}

\author{
P A McCormick, E A Sankey, F Cardin, A P Dhillon, N McIntyre, A K Burroughs
}

\begin{abstract}
Congestive gastropathy is a frequent cause of upper gastrointestinal haemorrhage in patients with portal hypertension. The pathogenesis is thought to involve venous congestion with gastric mucosal capillary dilatation. We studied the relation between gastric mucosal capillary dilatation, measured morphometrically, and endoscopic appearances in 74 patients with portal hypertension and 20 control subjects. We also investigated the frequency of gastric colonisation with Helicobacter pylori. Mucosal capillaries in patients were significantly dilated compared with control subjects $(p<0.001)$ but the degree of dilatation was not related to the severity of the endoscopic appearances. $H$ pylori was identified in 19 of $74(26 \%)$ patients but was not related to the severity of the endoscopic appearances. These results suggest that other factors in addition to mucosal venous and capillary congestion are important in the pathogenesis of endoscopic congestive gastropathy and that gastric colonisation with $\boldsymbol{H}$ pylori is unlikely to be one of these factors.
\end{abstract}

Congestive gastropathy is a frequent endoscopic finding in patients with portal hypertension and is believed to be responsible for approximately $20 \%$ of episodes of bleeding in these patients. The pathogenesis of the disorder is, however, controversial. McCormack et al' coined the term 'congestive gastropathy' and distinguished it histologically from inflammatory gastritis. They suggested that the endoscopic appearances were due to venous congestion and capillary dilatation in the gastric mucosa and submucosa. Most, ${ }^{2-4}$ but not all, ${ }^{5}$ authors agree that capillary dilatation is important in this condition. Morphometric analysis of capillary dilatation has rarely been performed in biopsy specimens from patients with portal hypertension. With the exception of a small subgroup of patients with very severe haemorrhagic gastritis, ${ }^{3}$ the degree of capillary dilatation seen in the gastric mucosa has not been evaluated with relation to the severity of the endoscopic appearances. The aim of this study was to assess the relation between mucosal capillary dilatation, measured morphometrically, and the endoscopic appearances. If venous and capillary congestion are closely related to the genesis of the endoscopic appearances, one would expect increased capillary dilatation in subjects with more severe endoscopic appearances. Alternatively, if mucosal congestion makes the mucosa more susceptible to damage from other agents ${ }^{6}$ or reduces its capacity to repair damage, the degree of capillary dilatation may not necessarily correlate closely with the endoscopic findings, as the most recent injury is the critical determinant of the endoscopic appearances.

A secondary aim of this study was to determine the prevalence of Helicobacter pylori colonisation in our patients. It has been suggested that there is a high prevalence of $H$ pylori colonisation in portal hypertension, particularly in patients with gastric erosions. ${ }^{7}$

\section{Patients and methods}

Seventy four patients with oesophageal varices underwent endoscopy on a total of 93 separate occasions, and multiple antral biopsy specimens were taken. Their mean age was 51.5 years (range 18-77 years) and 50 were men. Diagnoses included cirrhosis in 67 (30 alcoholic), portal vein block in four, schistosomiasis in two, and congenital hepatic fibrosis in one. Liver function was assessed on each occasion and graded according to Pugh's modification of Child's grading system, ${ }^{8}$ for cirrhotic patients $(51 \%$ were grade A, $\mathbf{4 0} \%$ grade $B, 9 \%$ grade $C$ ). Medications were also recorded. On 31 occasions patients were taking $\mathrm{H}_{2}$ antagonists, on 14 occasions sucralfate, on five occasions bismuth, and on one occasion antacids. No patients were taking nonsteroidal anti-inflammatory agents or $\beta$ blockers. Fifty nine per cent of patients had previously been treated with endoscopic sclerotherapy. Antral biopsy specimens were also taken from 20 control subjects, who had a mean age of 43.9 years (range 25-82 years) and of whom 14 were women. Control subjects were patients undergoing upper gastrointestinal endoscopy whose gastric biopsy specimens were found to be histologically normal. They included six patients with myalgic encephalomyelitis (ME syndrome) who had gastroscopy and biopsy as part of a research investigation. $H$ pylori was not identified in any of the control subjects.

At each endoscopy the severity of endoscopic gastropathy was graded using a modification of the classification previously employed by McCormack et al.' A four point scale was used as follows:

None $=$ normal appearances;

Mild=mosaic or snake skin appearance';

Moderate $=$ presence of erythema;

Severe $=$ presence of erosions or haemorrhagic gastritis.

The only change from the classification used by McCormack $e t a l$ is that we subdivided mild gastropathy into mild and moderate gastropathy. We put the snake skin or mosaic appearance into a category on its own in the belief that it may represent a less severe form of gastropathy than the presence of erythema or erosions and haemorrhages, or both. Endoscopy was performed using 


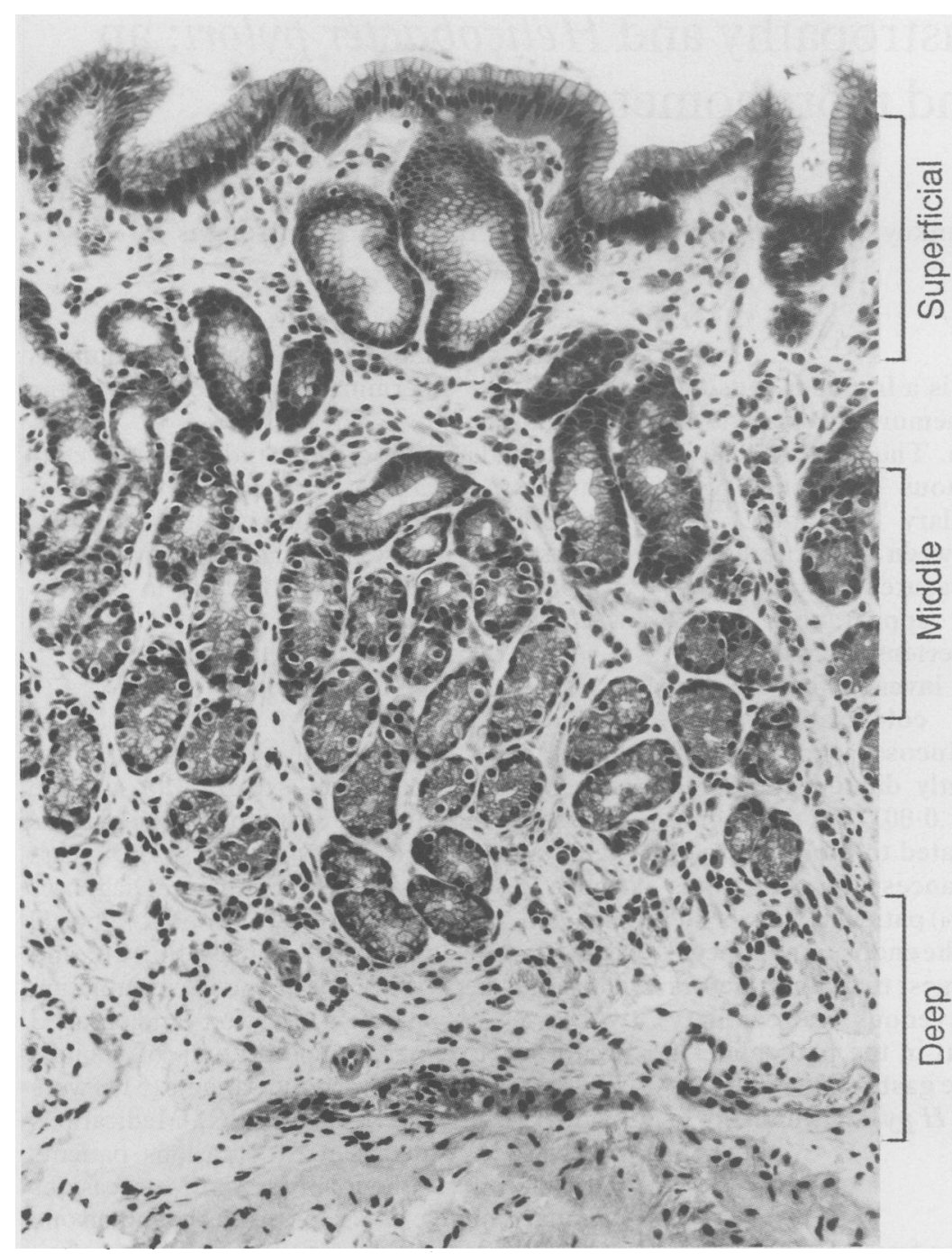

Figure 1: Haematoxylin and eosin stained section of gastric mucosa showing superficial, middle, and deep layers (original magnification $\times 40$ ).

Olympus K10, Q10, or XQ20 endoscopes and standard $5 \mathrm{~mm}$ biopsy forceps.

Two to four antral biopsy specimens were taken in each case and stained routinely with haematoxylin and eosin. $H$ pylori organisms were identified by careful examination of the stained biopsy specimens by two independent observers unaware of the endoscopic appearances. Histological gastritis was identified and graded semiquantitatively using a modification of Whitehead's criteria. ${ }^{10}$ 'Mild' gastritis was defined as superficial chronic gastritis. 'Moderate' gastritis was identified as a superficial gastritis with a moderate inflammatory infiltrate of plasma cells and lymphocytes, including small lymphoid aggregates but with no gland distortion. 'Severe' gastritis was defined as superfical active, or mild atrophic gastritis with a compact (intense) inflammatory infiltrate extending through the mucosa. In addition, neutrophils might or might not be present and there could be gland neck distortion or evidence of regeneration. On each occasion the cross sectional areas of 30 well orientated capillaries were measured using computer assisted image analysis (Logitec MD 20 densitometric system). Ten consecutive vessels were measured at each of three layers in the biopsy specimen (10 superficial, 10 middle, and 10 deep - Fig 1). Values were expressed as the mean (SEM) in $\mu \mathrm{m}^{2}$. Unless otherwise stated, all the figures and percentages refer to numbers of biopsy specimens rather than number of patients. The significance of differences between groups was tested using Student's $t$ test or the Mann-Whitney $U$ test as appropriate. The degree of correlation was checked using the Spearman rank correlation coefficient.

\section{Results}

\section{ENDOSCOPIC GASTROPATHY}

On all but eight occasions there was evidence of endoscopic gastropathy: mild $6(6 \%)$, moderate $61(66 \%)$, severe $18(19 \%)$. The severity of endoscopic gastropathy did not seem to be related to a history of previous endoscopic sclerotherapy $(\mathrm{p}=0.37)$ or to drug use: $\mathrm{H}_{2}$ antagonists $(p=0 \cdot 78)$, sucralfate $(p=0.83)$. Nor were the endoscopic appearances related to the presence or degree of histological gastritis (Table I) (coefficient of correlation $-0.007 \mathrm{p}=0.950$ ). The severity of endoscopic gastropathy was similar in patients with alcoholic cirrhosis compared with those with portal hypertension of other aetiologies. Mild, moderate, or severe gastropathy was noted at endoscopy in $7 \%, 70 \%$, and $16 \%$ of occasions in the alcoholics compared with $6 \%, 61 \%$, and $23 \%$ of occasions in the nonalcoholics. A similar pattern seen on histology as mild, moderate, and severe histological gastritis was noted in $55 \%, 20 \%$, and $9 \%$ of biopsy specimens from alcoholic patients compared with $25 \%, 37 \%$, and $16 \%$ respectively from nonalcoholic portal hypertensive patients.

\section{CAPILLARY DILATATION}

A total of 3140 capillaries were counted in the morphometric analysis. In six biopsy specimens from the portal hypertensive group and 13 from the control subjects there was insufficient deep layer mucosa in the specimens for analysis of capillary size in this layer.

TABLE I Prevalence of Helicobacter pylori $(H P)$ in relation to the degree of histological gastritis in biopsy specimens from patients with portal hypertension

\begin{tabular}{lllll}
\hline \multicolumn{5}{c}{ Histological gastritis } \\
\cline { 2 - 5 } & None & Mild & Moderate & Severe \\
\hline Capillary areas $\left(\mu \mathrm{m}^{2}\right)$ & $214(21)$ & $179(10)$ & $197(14)$ & $203(25)$ \\
$\begin{array}{l}\text { Mean }(\mathrm{SEM}) \\
\text { No HP +ve/total } \\
(\%)\end{array}$ & $0 / 13$ & $2 / 41$ & $11 / 27$ & $9 / 12$ \\
$\begin{array}{l}\text { Endoscopic gastropathy: } \\
\text { No (\%) }\end{array}$ & $(5)$ & $(41)$ & $(75)$ \\
None & $1 / 13$ & $2 / 41$ & $3 / 27$ & $1 / 12$ \\
Mild & $(8)$ & $(5)$ & $(11)$ & $(8)$ \\
& $0 / 13$ & $2 / 41$ & $2 / 27$ & $2 / 12$ \\
Moderate & $(0)$ & $(5)$ & $(7)$ & $(17)$ \\
Severe & $11 / 13$ & $28 / 41$ & $17 / 27$ & $5 / 12$ \\
& $(84)$ & $(68)$ & $(63)$ & $42)$ \\
& $1 / 13$ & $9 / 41$ & $5 / 27$ & $4 / 12$ \\
& $(8)$ & $(22)$ & $(19)$ & $(33)$ \\
\hline
\end{tabular}

Presence of $H$ pylori correlated with the severity of histological gastritis (Spearman correlation coefficient $0.57 ; p<0.001$ ).
Severity of histological gastritis was not correlated with severity of Severity of histological gastritis was not correlated with se
endoscopic gastropathy (Spearman correlation coefficient endoscopic gastropathy (Spearman correlation coefficient
$-0.007 ; p=0.95$ ) and did not significantly influence the degree of $-0.007 ; p=0.95)$ and did not significantly influence the degree
capillary dilatation in portal hypertensive patients. Severity of capillary dilatation in portal hypertensive patients. Severity of
histological gastritis did not significantly influence capillary histological
dilatation. 


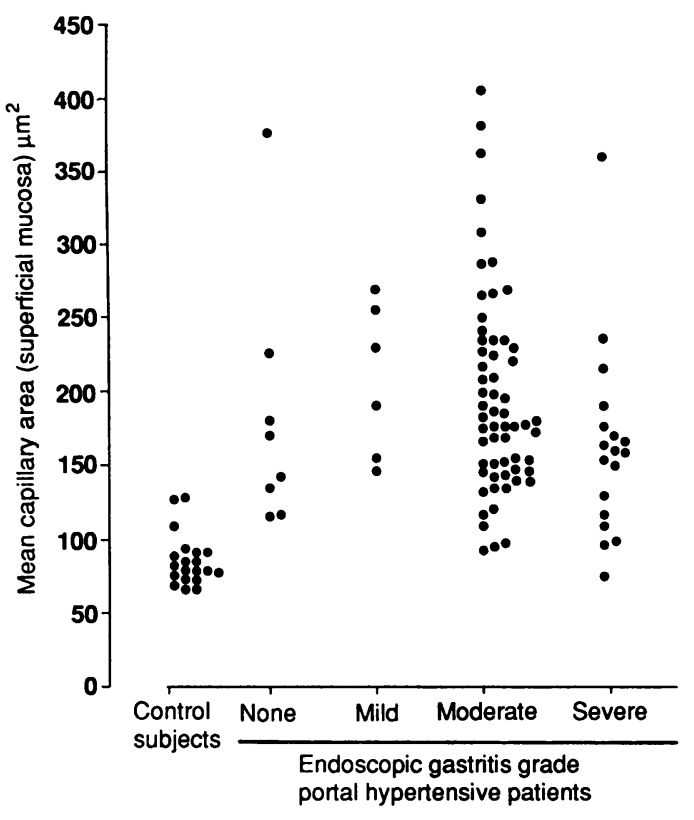

Figure 2: Mean capillary areas for individual patients in the superficial mucosal layer in controls and portal hypertensive patients. Capillary areas were significantly greater in four groups of portal hypertensive patients compared with controls $(p<0.001)$.

A summary of the results of the morphometric analysis is given in Table II. Superficial capillaries were significantly dilated in portal hypertensive patients with all grades of endoscopic appearance compared with control subjects: control subjects (mean (SEM) $87(4) \mu \mathrm{m}^{2}$ ); portal hypertensive patients with normal endoscopic appearances $\left(186(33) \mu \mathrm{m}^{2} ; \mathrm{p}<0.001\right)$, with mild gastropathy (210 (22) $\left.\mu \mathrm{m}^{2} ; \mathrm{p}<0.001\right)$, with moderate gastropathy (194 (9) $\left.\mu \mathrm{m}^{2} ; \mathrm{p}<0.001\right)$, and with severe gastropathy (174 (32) $\mu^{2}$; $\mathrm{p}<0.001$ ).

Individual results for mean capillary size in the superficial mucosa are shown in Figure 2. This is the layer which is most visible to the endoscopist. A similar pattern was seen in the middle mucosal layer but there was no significant difference between biopsy specimens from control subjects and patients with portal hypertension in the deep layer. There was no significant difference in capillary size between different grades of severity

TABLE II Capillary areas ( $\mu m^{2}$ values mean (SEM)) for control subjects and patients with portal hypertension according to endoscopic grade

\begin{tabular}{|c|c|c|c|c|c|}
\hline \multirow{2}{*}{$\begin{array}{l}\text { Mucosal } \\
\text { layer }\end{array}$} & \multirow[b]{2}{*}{ Control } & \multicolumn{4}{|c|}{ Endoscopic gastropathy } \\
\hline & & None & Mild & Moderate & Severe \\
\hline $\begin{array}{l}\text { Superficial } \\
\text { Middle } \\
\text { Deep }\end{array}$ & $\begin{array}{c}87(4) \\
125(8) \\
235(39)\end{array}$ & $\begin{array}{l}186(33)^{\star} \\
264(42)^{\star} \\
293(48)\end{array}$ & $\begin{array}{l}210(22)^{\star} \\
203(16)^{\star} \\
239(36)\end{array}$ & $\begin{array}{l}194(9)^{\star} \\
253(14)^{\star} \\
333(28)\end{array}$ & $\begin{array}{l}174(32)^{\star} \\
239(21)^{\star} \\
343(61)\end{array}$ \\
\hline
\end{tabular}

TABLE III Prevalence of Helicobacter pylori $(H P)$ in relation to the degree of endoscopic gastropathy

\begin{tabular}{lllll}
\hline & \multicolumn{4}{l}{ Endoscopic gastropathy } \\
\cline { 2 - 5 } & None & Mild & Moderate & Severe \\
\hline HP + ve/total & $3 / 8$ & $3 / 6$ & $12 / 61$ & $4 / 18$ \\
$(\%)$ & $(38)$ & $(50)$ & $(20)$ & $(22)$ \\
\hline
\end{tabular}

There is no significant correlation between the presence of $H$ pylori and the severity of endoscopic gastropathy (Spearman correlation coefficient $-0 \cdot 12 ; \mathrm{p}=0 \cdot 25)$. of endoscopic gastropathy in portal hypertensive patients at any of the three levels in the specimen. To allow for the possibility that the presence of histological gastritis may contribute to capillary dilatation, we compared mean capillary size in portal hypertensive patients with and without histological gastritis. The presence or severity of histological gastritis did not seem to influence the degree of capillary dilatation in any of the three mucosal layers. Figures for the superficial mucosal layer are summarised in Table I.

In addition, there were no significant differences in the degree of capillary dilatation in any of the three mucosal layers between patients who had and those who had not been treated previously with endoscopic sclerotherapy. The relevant figures; for the sclerosis and nonsclerosis groups respectively were mean (SEM) $196(10) \mu \mathrm{m}^{2} v 187(11) \mu \mathrm{m}^{2}(\mathrm{p}=0.85)$ for superficial mucosa; 255 (14) $\mu \mathrm{m}^{2} v 243$ (15) $\mu \mathrm{m}^{2}$ $(\mathrm{p}=0.91)$ for middle mucosa; and $338(20) \mu \mathrm{m}^{2} v$ $329(40) \mu \mathrm{m}^{2}(\mathrm{p}=0.72)$ for deep mucosa. The degree of capillary dilatation was similar in patients with alcoholic cirrhosis compared with subjects with other causes of portal hypertension. The figures for the superficial mucosa layer were mean (SEM) $188(10) \mu \mathrm{m}^{2}$ for biopsy specimens from alcoholic cirrhotic patients compared with 195 (11) $\mu \mathrm{m}^{2}$ for specimens from non-alcoholic patients.

\section{PREVALENCE OF HELICOBACTER PYLORI}

$H$ pylori was identified in 22 of 93 biopsy specimens $(23.7 \%)$ and in 19 of 74 patients (26\%). The presence of $H$ pylori was associated with histological gastritis (Table II), being absent in 13 biopsy specimens without histological gastritis and present in 9 of 12 specimens $(75 \%)$ showing severe histological gastritis (coefficient of correlation $0.57 \mathrm{p}<0.001$ ). In contrast, the presence of $H$ pylori was not closely associated with the severity of endoscopic gastropathy (Table III) (coefficient of correlation $-0 \cdot 12, \mathrm{p}=0 \cdot 25) . H$ pylori was identified in $38 \%$ of specimens from patients with normal endoscopic appearances and in $22 \%$ of those with severe endoscopic gastropathy.

\section{Discussion}

Our study confirms that gastric mucosal capillary dilatation is present in patients with portal hypertension as compared with control subjects. The degree of capillary dilatation did not seem to be influenced by the presence or absence of gastritis. Neither was the degree of capillary dilatation related to the severity of endoscopic appearances. Moreover the degree of endoscopic gastropathy and the degree of capillary dilatation did not seem to be influenced by prior sclerotherapy, as had been suggested previously.' These findings suggest that factors other than the degree of mucosal congestion may be important in the pathogenesis of the endoscopic changes.

Although the endoscopic appearances of congestive gastropathy are usually more noticeable in the fundus of the stomach, ${ }^{19}$ we chose to take antral biopsy specimens to avoid inadvertent biopsy of gastric varices and also to maximise the 
detection of $H$ pylori colonisation. We suspected initially that colonisation with $H$ pylori might be related to the severity of the endoscopic changes. Our results do not, however, support this hypothesis. $H$ pylori was identified in $26 \%$ of our patients, but was not related to the presence of endoscopic gastropathy. To identify $H$ pylori we used routine haematoxylin and eosin stained biopsy specimens. In our experience and that of others, ${ }^{112} 90 \%$ of $H$ pylori are detected by this method. The use of special stains might have increased the yield slightly ${ }^{11}$ but would not have altered the results significantly. The question as to whether $H$ pylori should be treated when identified has not been resolved in patients with peptic ulcer disease, ${ }^{13}$ and has not been evaluated in patients with portal hypertension. Given that the presence of $H$ pylori is unrelated to the endoscopic appearances, we treat only patients who also have dyspeptic symptoms.

We feel that the most likely explanation for the pathogenesis of the endoscopic changes in the gastric mucosa of patients with varices is that mucosal congestion may render the mucosa more prone to injury or reduce its capacity to repair injury. Support for this suggestion comes from work in animals showing an increased susceptibility of the portal hypertensive mucosa to injury from agents such as bile acids, aspirin, or alcohol. ${ }^{6}$ It is not known, however, whether results from the animal model used in these studies can be extrapolated to human portal hypertensive gastropathy ${ }^{14}$ and more work in this area is required. If the human portal hypertensive gastric mucosa is more prone to injury this would suggest that treatment could be aimed at the two mechanisms involved; either by reducing portal pressure with drugs ${ }^{15}$ or surgery ${ }^{16}$ or by trying to reduce gastric mucosal injury. The mechanisms of gastric injury in patients with portal hypertension must be more clearly elucidated in order to justify drug therapy in clinical practice. Trials of cytoprotective agents such as misoprostol may be worthwhile.

In summary, this study shows that the severity of the endoscopic appearances of congestive gastropathy in patients with varices does not correlate with either the degree of mucosal capillary dilatation or histological gastritis. This suggests that mucosal congestion is a necessary but not sole prerequisite for the development of the endoscopic appearances of congestive gastropathy. Other factors may play a role in the pathogenesis of gastric injury but colonisation with $H$ pylori is unlikely to be one of these.

We would like the thank Miss Doris Elliott for secretarial assistance. Dr Cardin was supported by the R Farimi Foundation for Gastroenterological Research.

1 McCormack TT, Sims J, Eyre-Brook I et al. Gastric lesions in portal hypertension: inflammatory gastritis or congestive gastropathy? Gut 1985; 26: 1226-32.

2 Foster PN, Wyatt JI, Bullimore DW, Losowsky MS. Gastric mucosa in patients with portal hypertension: prevalence of capillary dilatation and Campylobacter pylori. $\mathcal{f}$ Clin Pathol 1989; 42: 919-21.

3 Quintero E, Pique JM, Bombi JA et al. Gastric mucosal vascular ectasias causing bleeding in cirrhosis. A distinct entity associated with hypergastrinaemia and low serum entity associated with hypergastrinaemia and low serum

4 levels of pepsinogen 1. Gastroenterology 1987; 93: 1054-61. microcirculation in cirrhosis. Hepatology 1983; 3: 1008-12.

5 Corbishley CM, Saverymuttu SH, Maxwell JD. Use of endoscopic biopsy for diagnosing congestive gastropathy. $\mathcal{F}$ Clin Pathol 1988; 41 : 1187-90.

6 Sarfeh IJ, Somiman H, Waxman K et al. Impaired oxygenation of gastric mucosa in portal hypertension. The basis for increased susceptibility to injury. Dig Dis Sci 1989; 34: 225-8.

7 Paoluzi P, Piatroisuti A, Marchaggiano et al. Prevalence of Campylobacter pylori in cirrhotic patients with gastric Campylobacter pylori in cirrhotic patients with

8 Pugh RNH, Murray-Lyon IM, Dawson JL et al. Transection of the oesophagus for bleeding oesophageal varices. $\mathrm{Br} \mathcal{F}$ of the oesophagus 1973; 60: 646-9.

9 Papazian A, Braillon A, Dupas JL, Sevenet F, Capron JP. Portal hypertensive gastric mucosa; an endoscopic study. Gut 1986; 27: 1199-1203.

10 Whitehead R, Truelove SC, Gear MWL. The histological diagnosis of chronic gastritis in fibreoptic gastroscope biopsy specimens. F Clin Pathol 1972; 25: 1-11.

11 Hamilton-Dutoit SJ, Szeto ML, Dhillon AP, Pounder REP. The detection of Campylobacter pyloridis (CP) in gastric biopsies: a comparative study of histology and the "CLO biopsies: a comparative study of histol

12 Schnell GA, Schubert TT. Usefulness of culture, histology and urease testing in the detection of Campylobacter pylori. and urease testing in the detection of

13 Preston WL. Antibiotic therapy of duodenal ulcer? Hold off for now. Gastroenterology 1989; 97: 508-10.

14 Groszmann RJ, Colombata LA. Gastric vascular changes in portal hypertension. Hepatology 1988; 8: 1708-10.

15 Hosking SW, Kennedy HJ, Seddon E, Triger DR. The role of propranolol in congestive gastropathy of portal hypertension. Hepatology 1987; 7: 437-41.

16 Sarfeh IJ, Juler GL, Stemmer EA et al. Results of surgica management of haemorrhagic gastritis in patients with gastro-oesophageal varices. Surg Gynaecol Obstet 1982; 155: gastro-oesor
$167-70$. 